WellBeing International

WBI Studies Repository

2002

\title{
Chimpanzees (Pan troglodytes) Recognize Spatial and Object Correspondences Between a Scale Model and its Referent
}

Valerie A. Kuhlmeier

Yale University

Sarah T. Boysen

The Ohio State University

Follow this and additional works at: https://www.wellbeingintlstudiesrepository.org/acwp_asie

Part of the Animal Studies Commons, Comparative Psychology Commons, and the Other Animal Sciences Commons

\section{Recommended Citation}

Kuhlmeier, V. A., \& Boysen, S. T. (2002). Chimpanzees (Pan troglodytes) recognize spatial and object correspondences between a scale model and its referent. Psychological Science, 13(1), 60-63.

This material is brought to you for free and open access by WellBeing International. It has been accepted for inclusion by an authorized administrator of the WBI Studies Repository. For more information, please contact wbisr-info@wellbeingintl.org.

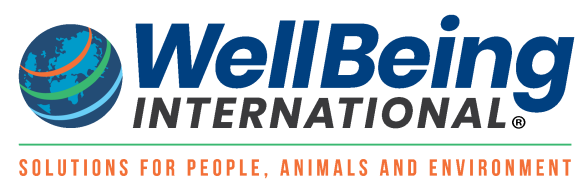




\title{
Chimpanzees (Pan troglodytes) Recognize Spatial and Object Correspondences Between a Scale Model and its Referent
}

\author{
Valerie A. Kuhlmeier ${ }^{1}$ and Sarah T. Boysen ${ }^{2,3,4}$ \\ ${ }^{1}$ Yale University \\ ${ }^{2}$ Ohio State University \\ ${ }^{3}$ Emory University \\ ${ }^{4}$ Bioqual, Inc.
}

\begin{abstract}
$\underline{\text { ABSTRACT }}$
In the present study, the contributions of spatial and object features to chimpanzees' comprehension of scale models were examined. Seven chimpanzees that previously demonstrated the ability to use a scale model as an information source for the location of a hidden item were tested under conditions manipulating the feature correspondence and spatial-relational correspondence between objects in the model and an outdoor enclosure. In Experiment 1, subjects solved the task under two conditions in which one object cue (color or shape) was unavailable, but positional cues remained. Additionally, performance was above chance under a third condition in which both types of object cues, but not position cues, were available. In Experiment 2, 2 subjects solved the task under a condition in which shape and color object cues were simultaneously unavailable. The results suggest that, much like young children, chimpanzees are sensitive to both object and spatial-relational correspondences between a model and its referent.
\end{abstract}

Understanding and effectively using a physical representation of space, such as a scale model or map, requires the ability to recognize the item-to-item correspondences and the spatial-relational correspondence between the representation and the real space to which it refers (Bluestein \& Acredolo, 1979; Huttenlocher, Newcombe, \& Vasilyeva, 1999; Marzolf, DeLoache, \& Kolstad, 1999). That is, one has to attend to the objects and spatial relations in one space and recognize their correspondence to the relations in another space. How and when humans come to understand these correspondences has been the focus of much work in developmental psychology (e.g., DeLoache, 1987, 1991; Huttenlocher et al., 1999; Liben \& Downs, 1989).

DeLoache has approached this question in her innovative research program exploring children's ability to understand the representational nature of a scale model (e.g., DeLoache, 1987; DeLoache, Kolstad, \& Anderson, 1991; Marzolf et al., 1999). She and her colleagues have found that after witnessing a miniature item being hidden in a scale model of a room, 3-year-olds can locate a full-size item hidden in the analogous location in the real room. There are potentially three ways that children might determine 
where the item is hidden and solve this task. First, they can recognize solely the correspondence between the individual hiding sites in the model and the room (object-landmark correspondence). Second, they can attend only to the correspondence in the spatial layout among the sites in the model and room (relational-geometric correspondence). Or third, they can recognize and use both types of correspondence.

Marzolf et al. (1999) have shown that children are sensitive to both the relational and the object correspondence between the model and room. They found that 3-year-olds had difficulty solving the scale-model task if the spatial relations among the objects in the model did not match the relations among objects in the room. That is, the absence of relational cues posed a problem for young children as they tried to map from model to room. It is important to note, however, that in this study, the incongruency between the spatial relations in the model and room was not the only factor causing children to have difficulty recognizing the model-room correspondence. Children had difficulty only under a condition in which relational correspondence was not present and a shortened version of the pretest introduction that was usually included in the orientation was given. Children who were tested under a condition in which the full orientation was given, yet spatial relations were incongruent, were able to solve the task by relying on object correspondence alone. Thus, the authors concluded that relational correspondence is recognized, but is one of many factors influencing performance. Consistent with this interpretation is the demonstration that when 3-year-olds are tested in a model task in which the hiding sites are perceptually identical (and, thus, object cues are unavailable), there is a decrement in performance compared with the standard conditions during which hiding sites are perceptually unique (Blades \& Cooke, 1994; Marzolf, 1996).

The ability of 3-year-olds to recognize relational correspondence is further supported by Huttenlocher et al. (1999). In their study, the majority of 3-year-old children demonstrated scaling ability, a requisite to understanding correspondence of spatial relations. These children were able to understand how the distance on a map corresponded to a distance in the real world. Together, the model and map studies demonstrate that by the age of 3 , children recognize when two spaces correspond in terms of the spatial relations among their components.

The present study addressed the question of whether animals, adept at using landmarks and geometric relations between landmarks in navigation (e.g., Cheng, 1986; Gouteux, Thinus-Blanc, \& Vauclair, in press; Gouteux, Vauclair, \& Thinus-Blanc, 1999; Spetch et al., 1997), can understand representational depictions of these relations such as maps or scale models. Until recently, it was not known if a nonhuman species can detect any correspondence between a physical representation of space and its referent. In two studies (Kuhlmeier \& Boysen, in press-a; Kuhlmeier, Boysen, \& Mukobi, 1999), we found that chimpanzees were able to solve a scale-model task that was similar to DeLoache's (e.g., 1987). In our original scale-model study (Kuhlmeier et al., 1999), the subjects observed as a miniature juice bottle was hidden within a scale model of an outdoor enclosure. The chimpanzees were then allowed to search the full-size enclosure to find a full bottle of juice that was hidden in the analogous location. Three, and under some conditions 4 , out of the 7 chimpanzees tested were able to solve this task and find the juice on the first search attempt. With this procedure, however, the juice reward could ultimately be obtained on every trial by simply continuing to search the other sites. We then modified the task by hiding an empty bottle of juice within the enclosure, and making reward contingent upon correct search choices (Kuhlmeier \& Boysen, in press-a). Under this procedure, the performance of the previously unsuccessful chimpanzees improved to significantly above chance. These studies demonstrated that, much like the children who were successful in DeLoache's experiments, the chimpanzees were able to solve the task, and to do so without prior training. 
What remains unknown is how the chimpanzees solved the task. That is, were they relying on object correspondences, relational correspondences, or some combination of both? Successful recognition of both kinds of cues would imply that chimpanzees and young children solve the scale-model task in a similar manner. Thus, testing adult chimpanzees might provide evidence that a nonverbal species has cognitive abilities similar to those seen to emerge in 3-year-old children for reasoning about two spaces in terms of their spatial relations. In the present experiments, we examined the influence of spatial and object correspondence on chimpanzees' comprehension of scale models. Experiment 1 examined the influence of these cues by systematically removing position (spatial) and color and shape (object) cues, and in Experiment 2, solving the scale-model task was solely dependent on the recognition of the spatialrelational correspondence between the model and enclosure.

\section{EXPERIMENT 1}

In Experiment 1, chimpanzees were tested under three conditions that manipulated the object cues of color and shape and the spatial-relational cue of position. It should be noted that each of the chimpanzee subjects had been pilot-tested under these conditions a year prior to the present study (see Kuhlmeier, 2000, and Kuhlmeier \& Boysen, in press-b). However, the trials were conducted using a procedure, detailed in the introduction, that has since been shown to result in suboptimal performance by some of the chimpanzees (Kuhlmeier \& Boysen, in press-a; Kuhlmeier et al., 1999). Consequently, performance for all chimpanzees in these pilot experiments was not significantly different from chance. The present experiment tested the chimpanzees using a slightly modified procedure.

\section{Method}

Subjects

Seven adult chimpanzees who participated in previous experiments using the scale-model task (Kuhlmeier \& Boysen, in press-a; Kuhlmeier et al., 1999) served as subjects in the current study. The group consisted of 3 females (Abby, 26 years; Sarah, 40 years; and Sheba, 18 years) and 4 males (Bobby, 13 years; Darrell, 19 years; Digger, 11 years; and Kermit, 19 years). All but 2 of the chimpanzees (Abby and Digger) also had previous experience on a range of cognitive tasks (e.g., Boysen, Berntson, \& Mukobi, 2001; Boysen, Mukobi, \& Berntson, 1999).

\section{Materials}

The materials were similar to those we have described elsewhere (Kuhlmeier et al., 1999). All testing occurred in an outdoor enclosure $(3.25 \mathrm{~m} \times 5.48 \mathrm{~m})$ that was made visually inaccessible from adjacent enclosures by canvas tarps hung on the mesh walls that separated the enclosures. The hiding sites in the model and room were perceptually unique plastic toys familiar to all of the subjects, and included a brown barrel, a large wooden cable spool, a pink children's slide, and a blue trash-can lid. A 1:7 scale model of the outdoor area that contained miniature versions of the hiding sites was presented to individual subjects. A full-size empty plastic juice bottle was hidden in the outdoor enclosure in a different location on each trial, and a miniature version of the bottle was used for the hiding event in the model.

\section{Procedure}

In all conditions, a subject watched from an overhead chute as the experimenter placed a miniature version of a plastic juice bottle in one of the four hiding sites in the scale model. Next, the subject was given access to the outdoor enclosure, where an empty, full-size plastic juice bottle had been hidden in the analogous site. If the animals chose the correct site and retrieved the empty bottle on their first attempt, they were encouraged (through pointing, gesturing, and vocal requests) to trade the empty bottle 
for a full bottle of juice, which they were permitted to drink. ${ }^{1}$ If they chose an incorrect site, they returned to the chute for the next trial. Each chimpanzee completed eight trials (two trials per hiding site and usually two trials per day) in each of three conditions. To control for order effects, we pseudorandomly assigned condition order across all subjects.

\section{Conditions}

In the color + shape condition, the spatial arrangements of the model and enclosure differed. On a given trial, for example, a chimpanzee might observe as the miniature bottle was hidden under the blue lid in the front left position of the model, but the comparable blue lid in the enclosure, which contained the hidden empty bottle, was located in the back left position. Consequently, individual color and shape cues of the objects were present, but spatial cues were discordant. Finding the bottle would indicate that the chimpanzee could recognize and rely on the correspondence between the color and shape of the miniature and full-size hiding sites, and thus did not require spatial-relational information.

In the position + shape condition, the hiding sites in the model and enclosure were configured in the same spatial arrangement. However, the objects at the analogous sites were not the same color. For example, if the bottle was hidden under the pink lid in the back left position of the model, the correct hiding site in the enclosure was the blue lid in the back left position. Thus, there was congruency between spatial cues and object shape cues in the model and the enclosure, but the object color cues did not correspond. A successful search under this condition would imply that the chimpanzee was able to recognize the hiding site's shape, the spatial-position correspondences between the model and the enclosure, or both.

In the position + color condition, the hiding sites in the model and enclosure were in the same spatial arrangement, and colors of the individual hiding sites were consistent. However, the objects at the sites differed in shape. For example, if the hiding event in the model occurred in the rectangular, blue slide at the back left position of the scale model, the correct hiding site in the enclosure was the round, blue lid at the back left position in the enclosure. Thus, success under this condition would imply that the chimpanzee was able to recognize the hiding site's color, the spatial-position correspondences between the model and the enclosure, or both.

\section{Statistical analyses}

For both Experiments 1 and 2, only bottle retrievals that occurred on the first search attempt were considered correct responses. All trials were videotaped, and the chimpanzees' responses were also recorded by hand by a second experimenter who was naive to the hiding site in the model for each trial. ${ }^{2}$ Because of the modest $N$ and to avoid violation of normality assumptions, we used nonparametric tests for all analyses, with alpha levels set at .05. To analyze individual responses, we adopted the following criterion. Performance was considered successful if a subject was correct on five out of eight trials. This criterion was adopted because five out of eight successful trials, with chance level equal to .25, constitutes a reliable, above-chance performance (binomial test, $p<.05$ ).

\section{Results and Discussion}

Under each condition, all but 2 of the chimpanzees (Abby and Digger) were able to find the hidden empty bottle at a level above chance. In each condition, each of these chimpanzees had five or more correct retrievals, $p<.05$ (Table 1). Similarly, at the group level, the chimpanzees were able to solve the task under all three conditions (sign tests, all medians $\geq 5$ correct retrievals, $p<.05$ ). Mann-Whitney tests revealed no sex differences in any condition, and Friedman's test revealed no difference in group performance among the conditions. There was also no difference in performance between the first and second half of trials, indicating consistent performance across the eight trials. In summary, under 
conditions in which no spatial cues were present (color + shape), under conditions in which no object color cues were available (position + shape), and under conditions in which no object shape cues were available (position + color), the chimpanzees were able to solve the scale-model task.

The successful performance in all three conditions suggests that the chimpanzees recognized both the object and the spatial-relational correspondences between the model and the enclosure. In fact, the chimpanzees did not have difficulty switching from one condition to the other. That is, after completing eight trials under one condition, they frequently were able to correctly solve the first trial performed under the next condition (median number of correct first trials across chimpanzees was two first trials out of the three). Thus, the chimpanzees responded with flexibility to modifications of the object and spatial correspondences between the model and enclosure, suggesting that they were prepared to use these cues to solve the task.

Table 1. Number of correct responses for each chimpanzee in Experiments 1 and 2

\begin{tabular}{|lcccc|}
\hline & \multicolumn{4}{c|}{ Condition } \\
\cline { 2 - 5 } Subject & Color + shape & Position + shape & Position + color & Position only \\
\hline Abby & 4 & 3 & 3 & 2 \\
Sarah & 6 & 7 & 5 & 3 \\
Sheba & 7 & 6 & 5 & 4 \\
Bobby & 7 & 5 & 5 & 4 \\
Darrell & 6 & 6 & 7 & 6 \\
Digger & 2 & 3 & 3 & 3 \\
Kermit & 5 & 5 & 5 & 6 \\
\hline
\end{tabular}

Note. Five successful trials out of eight, with chance level equal to .25 , constitutes a reliable, above-chance performance (binomial test, $p<.05$ ).

\section{EXPERIMENT 2}

The chimpanzees were next tested under a condition in which object cues (color and shape) were simultaneously uninformative about the location of the hidden bottle, and thus, the subjects had to rely entirely on spatial cues. That is, the four hiding sites were identical in appearance, and success on the task required recognition of the relational correspondences between the model and its corresponding fullsize space.

\section{Method}

The procedures were similar to those of Experiment 1; however, in Experiment 2, the enclosure contained four identical red plastic tubs that served as hiding sites, and the scale model contained miniature versions of the sites (position-only condition). Each chimpanzee received eight randomly ordered test trials (two trials per hiding site and typically two trials per day).

\section{Results and Discussion}

Two of the chimpanzees (Darrell and Kermit) were able to find the empty bottle on the first search attempt at levels significantly above chance (each had six correct retrievals, $p<.05$; see the position-only condition in Table 1). At the group level, the chimpanzees' performance was above chance (sign test, median $=4$ correct retrievals, $p<.05$ ), and Mann-Whitney tests revealed no sex differences in any condition. Thus, when object cues were removed and only spatial-relational cues reliably offered 
information as to the location of the hidden bottle, the chimpanzees as a group were still able to solve the task. However, the individual performance of 3 subjects who were successful in Experiment 1 (Sarah, Sheba, Bobby) showed deterioration, and the performance of all the subjects except Darrell and Kermit dropped relative to their performance in previous studies in which both object and spatial cues were available (i.e., Kuhlmeier \& Boysen, in press-a; Kuhlmeier et al., 1999). This suggests that these test conditions represented a more difficult task for some of the chimpanzees. On the whole, however, the results suggest that chimpanzees can recognize the spatial-relational correspondence between the hiding sites in the model and the real enclosure. Indeed, for 2 of the subjects, performance was no different from when object cues were available. Yet the results are similar to those with 3-year-old children (Blades \& Cooke, 1994; Marzolf, 1996) in that for some chimpanzees this correspondence might not be as readily appreciated when object cues are absent as when they are present.

\section{GENERAL DISCUSSION}

The present experiments suggest that chimpanzees recognize both the object and the relational correspondences between a scale model and its referent, and can use these correspondences as sources of information for subsequent navigation within their environment. As a group, the chimpanzees were successful under all three conditions presented during Experiment 1, and at the individual level, 5 of the 7 chimpanzees performed significantly above chance level. That is, when no spatial cues were present (color + shape), when no object color cues were available (position + shape), and when no object shape cues were present (position + color), the chimpanzees were able to solve the scale-model task. It is important to note that successful searching under the color + shape condition demonstrated that chimpanzees were able to solve the task in the absence of spatial-relational cues and, thus, rely solely on object correspondences. However, these results do not necessarily imply that the chimpanzees did not use relational cues when they were present. Indeed, their success under the other two conditions of Experiment 1 suggests that they could also solve the task when object correspondences were not consistent, yet relational cues remained.

A possible argument against this interpretation is that the chimpanzees were solving the task under the position + color and position + shape conditions by relying on the one aspect of the object that remained congruent between the model and enclosure (color only for position + color and shape only for position + shape), and disregarding the spatial cues that were also available. This is unlikely for two reasons. First, the flexible manner in which the chimpanzees were able to switch from one condition to the other suggests that they also may have been relying on spatial cues. For example, a subject that completed testing under the position + shape condition followed by the position + color condition could not likely have shown immediate success in the latter condition if he or she were relying only on object cues. However, if the subject could flexibly rely on spatial cues as well, the observed rapid transfer of successful performance under the new condition would be possible. Second, as a group, the chimpanzees performed significantly above chance in Experiment 2, when only spatial cues provided information as to the location of the hidden bottle, suggesting that the chimps did recognize the relational correspondence between the two spaces. Indeed, 2 subjects performed above chance at the individual level. It is important to note that the performance of these 2 chimpanzees differed from that of 3-year-old children tested in a similar task (Blades \& Cooke, 1994; Marzolf, 1996). Whereas 3-year-olds showed a performance decrement when object cues were completely absent, these 2 chimpanzees did not. This difference implies that there may be species differences in the sensitivity to relational correspondence in the scale-model task. However, 4 of the chimpanzees did show a decrement in performance, suggesting that further study is necessary to explore this possible species difference.

On the whole, however, the chimpanzees' demonstrated sensitivity to both the object and the relational correspondences between the model and its referent appears very similar to the skills that emerge in 
children by the age of 3 years. In search tasks using scale models and maps, children have demonstrated sensitivity to both of these cue sources (e.g., DeLoache et al., 1991; Huttenlocher et al., 1999; Marzolf et al., 1999). The present study shows that this ability extends to a nonverbal species, indicating that the recognition of both the object and the relational correspondences between a physical representation of space and its referent does not require spatial language. It will be informative to discover the limits on chimpanzees' sensitivity to these cues, as well as instances in which one cue type may be more salient than the other. Additionally, examining when these abilities emerge in the young chimpanzee, and the types of spatial representations that chimpanzees may be able to understand, will help to elucidate the developmental trajectory of these capacities in this species and allow for further comparisons with human children.

\section{Notes}

${ }^{1}$ The chimpanzees had many years of experience with trading objects in their enclosure for food rewards, and none had difficulty generalizing to the current task.

${ }^{2}$ For purposes of generating data on interobserver reliability, a third experimenter who was also naive to the hiding sites viewed all videotapes and recorded the chimpanzees' responses. These data were then compared with data collected by the second experimenter. Cohen's Kappa statistic $(K)$ was used as a measure of reliability between observers. Interobserver measures indicated strong agreement between observers for both experiments $(K \geq .98)$.

\section{Acknowledgments}

We would like to thank Vladimir Sloutsky, Nora Newcombe, and Judy DeLoache for helpful comments. Kimberly Mukobi, Kelli Barritt, Carmen Owens, and graduate and undergraduate students assisted in the daily care of the chimpanzees at The Ohio State University Chimpanzee Center. The experiments reported in this article were conducted while the first author was at The Ohio State University and fulfilled part of the requirements for her doctoral degree.

\section{REFERENCES}

Blades, M., \& Cooke, Z. (1994). Young children's ability to understand a model as a spatial representation. Journal of Genetic Psychology, 115, 201-218.

Bluestein, N., \& Acredolo, L. (1979). Developmental changes in map-reading skills. Child Development, 50, 691-697.

Boysen, S.T., Berntson, G.G., \& Mukobi, K.M. (2001). Size matters: Impact of item size and quantity on array choice by chimpanzees. Journal of Comparative Psychology, 115, 106-110.

Boysen, S.T., Mukobi, K.L., \& Berntson, G.G. (1999). Overcoming response-bias using symbolic representations of number by chimpanzees (Pan troglodytes). Animal Learning and Behavior, 27, 229-235.

Cheng, K. (1986). A purely geometric module of the rat's spatial representation. Cognition, 23, 149-178.

DeLoache, J.S. (1987). Rapid change in the symbolic functioning of very young children. Science, 238, 1556-1557.

DeLoache, J.S. (1991). Symbolic functioning in very young children: Understanding of pictures and models. Child Development, 62, 736-752. 
DeLoache, J.S., Kolstad, D.V., \& Anderson, K.N. (1991). Physical similarity and young children's understanding of scale models. Child Development, 62, 111-126.

Gouteux, S., Thinus-Blanc, C., \& Vauclair, J. (in press). Young rhesus monkey's spatial reorientation: The use of geometric and featural information. Journal of Experimental Psychology: General.

Gouteux, S., Vauclair, J., \& Thinus-Blanc, C. (1999). Reaction to spatial novelty and exploratory strategies in baboons. Animal Learning and Behavior, 27, 323-332.

Huttenlocher, J., Newcombe, N., \& Vasilyeva, M. (1999). Spatial scaling in young children. Psychological Science, 10, 393-398.

Kuhlmeier, V.A. (2000). Use of a physical representation of space by chimpanzees (Pan troglodytes): Comparative perspectives on scale model comprehension. Unpublished doctoral dissertation, The Ohio State University, Columbus.

Kuhlmeier, V.A., \& Boysen, S.T. (in press-a). The effect of response contingencies on scale model task performance by chimpanzees (Pan troglodytes). Journal of Comparative Psychology. Kuhlmeier, V.A., \& Boysen, S.T. (in press-b). Representational capacities for pretense with scale models and photographs in chimpanzees (Pan troglodytes). In R. Mitchell (Ed.), Pretending in animals and humans. Cambridge, England: Cambridge University Press.

Kuhlmeier, V.A., Boysen, S.T., \& Mukobi, K.L. (1999). Scale model comprehension by chimpanzees (Pan troglodytes). Journal of Comparative Psychology, 113, 396-402.

Liben, L., \& Downs, R. (1989). Understanding maps as symbols. In H.W. Reese (Ed.), Advances in child development and behavior (Vol. 22, pp. 145-201). New York: Academic Press.

Marzolf, D.P. (1996). Relational mapping in symbolic tasks by young children. Unpublished doctoral dissertation, University of Illinois, Urbana-Champaign.

Marzolf, D.P., DeLoache, J.S., \& Kolstad, D.V. (1999). The role of relational similarity in young children's use of a scale model. Developmental Science, 2, 296-305.

Spetch, M.L., Cheng, K., MacDonald, S.E., Linkenhoker, B.A., Kelly, D.M., \& Doerkson, S.R. (1997). Use of landmark configuration in pigeons and humans: II. Generality across search tasks. Journal of Comparative Psychology, 111, 14-24. 